\title{
Abelian and Non-abelian Hall Liquids and Charge Density Wave: Quantum Number Fractionalization in One and Two Dimensions
}

\author{
Alexander Seidel and Dung-Hai Lee \\ Department of Physics, University of California at Berkeley, Berkeley, CA 94720, USA and \\ Material Science Division, Lawrence Berkeley National Laboratory,Berkeley, CA 94720, USA.
}

(Dated: September 26, 2018)

\begin{abstract}
Previously we have demonstrated that, on a torus, the abelian quantum hall liquid is adiabatically connected to a charge density wave as the smaller dimension of the torus is varied. [1] In this work we extend this result to the non-abelian bosonic Hall state. The outcome of these works is the realization that the paradigms of quantum number fractionalization in one dimension (polyacetylene) 2] and two dimensions (fractional quantum Hall effect) [3] are in fact equivalent.
\end{abstract}

Quantum number fractionalization is a subject of considerable interest in condensed matter physics recently. Two well-known examples where such phenomenon occurs are (1) the soliton in a one-dimensional (1D) charge density wave 2], and (2) the quasiparticles in the fractional quantum Hall effect 3]. Usually they are regarded as distinct mechanisms of charge fractionalization.

In a recent paper, Seidel et al have shown that when placed on a $L_{1} \times \infty$ torus, the $\nu=1 / 3$ fractional quantum Hall liquid is adiabatically connected to a period-three charge density wave when $L_{1}$ is varied 1 ]. In a subsequent work the same conclusion was reached independently [4]. Such continuity is very powerful in identifying discrete quantum numbers, since the latter cannot change during an adiabatic process. For example, the topological three fold-degeneracy in the large $L_{1} / l_{B}$ limit $\left(l_{B}\right.$ is the magnetic length) is adiabatically related to the three charge density wave patterns at small $L_{1} / l_{B}$. Moreover, the charge $1 / 3$ quasiparticles at large $L_{1} / l_{B}$ are evolved from the Su-Schrieffer domain walls at small $L_{1} / l_{B}[1]$.

The above results are based on a $2 \mathrm{D} \rightarrow 1 \mathrm{D}$ mapping discussed in Ref. [5, [6]. Specifically, in the Landau gauge, and after projection to the lowest Landau level, the pseudopotential Hamiltonian [7], for which the Laughlin state is the exact ground state, becomes a center-of-mass position conserving pair hopping model on a 1D lattice 1,5$]$. The torus parameter $L_{1} / l_{B}$ becomes the hopping range. Within this model it can be shown that for all non-zero $L_{1} / l_{B}$ there is an energy gap separating the three degenerate ground states from the excited states. In addition, each ground state has a different center-of-mass position, and exhibits a non-zero charge density wave order as long as $L_{1} / l_{B}$ is finite [1, 8]. Finally, due to the simultaneous conservation of the center of mass position and momentum, all eigenstates are at least three-fold degenerate.

In this paper, we apply the same mapping to study the bosonic non-abelian quantum Hall state at filling factor $\nu=1[9]$. Our motivations are as follows. First, there is a general argument due to Oshikawa 10, which says that at filling factor $\nu=p / q$, if a system possesses an excitation gap, then the ground state must be at least q-fold degenerate on a (d-dimensional) torus. From this point of view, one expects that in the absence of hidden symmetry, the ground state for a gapped $\nu=1$ system should be non-degenerate. However, based on analyzing the ground state wavefunction, it was shown that the gapped non-Abelian bosonic Hall state is three-fold degenerate on torus 11, 12]. Second, we are curious whether the adiabatic connection in the $\nu=1 / 3$ abelian quantum Hall state also holds true for the non-abelian case.

In the infinite 2D plane the bosonic Pfaffian state $[9]$

$$
\Psi=\operatorname{Pf}\left[\frac{1}{z_{i}-z_{j}}\right] \prod_{(i j)}\left(z_{i}-z_{j}\right) \exp \left[-\sum_{k}\left|z_{k}\right|^{2} / 4\right] .
$$

is the unique ground state wavefunction of the following three-particle pseudopotential Hamiltonian at $\nu=1$

$$
H=\sum_{(i j k)} \delta\left(z_{i}-z_{j}\right) \delta\left(z_{i}-z_{k}\right) .
$$

When the Pfaffian state is generalized to toroidal geometry 11, 12], a threefold degeneracy emerges. As emphasized in the beginning, unlike the Abelian quantum Hall state, this degeneracy cannot be attributed to different center-of-mass quantum numbers.

Following our procedure in Ref. [1] we first establish a simple picture for this degeneracy by making the smaller dimension of the torus much smaller than the magnetic length $l_{B}$. To this end, we express the Hamiltonian of Eq. (2) in the Landau gauge basis of lowest Landau level of a $L_{1} \times L_{2}$ torus and obtain $H=\sum_{R} Q_{R}^{\dagger} Q_{R}$, where

$Q_{R}=\sum_{m+n+p=3 R \bmod N} f(R-m, R-n, R-p) c_{m} c_{n} c_{p}(3)$ and $f(a, b, c)=\kappa \sum_{s, t} \exp \left[-\left((a+s N)^{2}+(b+t N)^{2}+(c-\right.\right.$ $\left.(s+t) N)^{2}\right) / 2$ ]. In the above $m, n, p$ runs over the index of the lowest Landau level orbitals. One may identify each orbital with a site on a 1D ring. The total number of lattice site, $N$, is equal to the number of magnetic flux quanta piercing through the surface of the torus,i.e., $N=L_{1} L_{2} / 2 \pi l_{B}^{2}$. The operator $c_{n}$ annihilates a boson on the $n$-th site. Eq. (3) describes center-of-mass conserving triplet hopping. The only parameter, $\kappa$, of this Hamiltonian, $\kappa=2 \pi l_{B} / L_{1}$, sets the hopping range. 

(a) 0202020202020202020202020202020202
(b) 2020202020202020202020202020202020
(c) 1111111111111111111111111111111111
(d) 0202020202020202010202020202020202
(e) $0202020|1111111111111111111| 02020202$
(f) $11111|0202020202020202020| 1111111111$
(g) 02020|1111111|020202020|1111111|020202
(h) $02020 \mid 111111020202020201111111020202$
(i) 111111111111020202020201111111111111
(j) 111111111111|02020202020|11111111111

FIG. 1: The large $\kappa$ limits of the ground state (a)-(c), 2hole excited states (d)-(f) and (i),(j), and 4-hole excited state $(\mathrm{g}),(\mathrm{h})$. A vertical segment indicated the position of a charge $\Delta Q=1 / 2$ domain wall, see text.

Symmetry The Hamiltonian in Eq. (3) not only commutes with the lattice translation operator, $T$, but also commute with the operator

$$
U=\exp \left[2 \pi i / N \sum_{n} n c_{n}^{\dagger} c_{n}\right]
$$

We identify $U$ as the center-of-mass position operator of the ring. For filling factor $\nu=p / q$ it is simple to prove that $U T=e^{i 2 \pi p / q} T U$. Thus for $\nu=1$ the two operators $T$ and $U$ commute. As the result we can label any energy eigen state by three quantum number, i.e., the eigenvalue with respect to $H, U$ and $T$, i.e $|E, u, t\rangle$ where $H|E, u, t\rangle=E|E, u, t\rangle, U|E, u, t\rangle=\exp (i u)|E, u, t\rangle$, and $T|E, u, t\rangle=\exp (i t)|E, u, t\rangle$.

In addition to the above symmetries, Eq. (3) has the remarkable property that it is "self-dual" under Fourier transformation [1]. That is, when the model is reexpressed in momentum space, which is a lattice of discrete momenta, $2 \pi n / N$, one obtains a Hamiltonian which is identical to the real space one, except for an inversion of the parameter $\kappa \rightarrow \tilde{\kappa}=2 \pi / \kappa N$. Thus a Hamiltonian at $\kappa$ in the real space representation is identical to the Hamiltonian at $\tilde{\kappa}$ in momentum space. Consequently, the spectrum of Eq. (3) at $\kappa$ is identical to that at $\tilde{\kappa}=2 \pi / \kappa N$. Since the quantum number $u$ and $t$ interchanges upon going from the real to the momentum space and vice versa, it follows that all states at $\kappa$ that have quatum number $Q=(u, t)$ are degenerate with states at $\tilde{\kappa}$ with quantum number $Q=(t, u)$.

The large $\kappa$ limit Since for any $L_{1} / l_{B}$, the three Pfaffian ground states 11, 12 are zero energy eigenstates of Eq. (21), it follows that for any $\kappa$ there must be three ground states each annihilated by all the operators $Q_{R}$. We will now solve Eq. (3) in the limit $\kappa \gg 1$. By keeping the two largest hopping amplitudes we obtain

$$
H \approx \kappa^{2} \sum_{n}\left[\left(c_{n}^{\dagger}\right)^{3}\left(c_{n}\right)^{3}+\exp \left(-\kappa^{2} / 3\right)\left(c_{n}^{\dagger}\right)^{2} c_{n \pm 1}^{\dagger}\left(c_{n}\right)^{2} c_{n \pm 1}\right]
$$

Such a Hamiltonian penalizes states that have three particles occupying the same site, or three particles occupying two adjacent sites. All other terms in the Hamiltonian are exponentially smaller than the ones retained. The three zero energy states of Eq. (5) are shown in Fig.1(a)(c). Among them the two states in panel (a) and (b) break the translational symmetry. The third state shown in panel (c) is translationally invariant. These states will be referred to as (02)-, (20)- and (11)-state from now on. Thus, as in the abelian-case [1], we do obtain a simple pictures for the ground states in the large $\kappa$ limit. The primary question to be answered now is whether the low energy physics at large $\kappa$ is in fact adiabatically connected to the limit $\kappa \rightarrow 0$, as we argued in the abelian case.

The degeneracy Before we turn to this central issue, we first highlight some crucial differences between the abelian and the non-abelian problem. First, while in both cases the correct ground state degeneracy becomes manifest in the large- $\kappa$ limit, for the abelian case this degeneracy is dictated by (i) translation invariance, (ii) conservation of the center-of-mass position, and (iii) the non-commutivity between $T$ and $U$. Thus it holds true at any value of $\kappa$. Theis argument is no longer valid in the present case, because $T$ and $U$ commute. Furthermore, all of the ground state in the abelian case are related by a simple lattice translation, and are thus energetically equivalent. Theis is not true in the present case, since at large $\kappa$ only two of the ground states are related by translation. Indeed, the (11)-state appears as special when compared to the (02)- and (20) states, and it seems that a nearest neighbor density-density interaction could lift the threefold degeneracy. While this is true at large $\kappa$, we believe that such lifting of degeneracy will become exponentially small as $\kappa \rightarrow 0$, and the threefold degeneracy will become robust at small $\kappa$. This is because, as we shall show later, the order parameter associated with the (02) and (20) state exponentially vanishes as $\kappa \rightarrow 0$.

We now show that assuming the existence of adiabatic continuity (to be shown below), the 3 -fold ground state degeneracy at any $\kappa$ can be explained as a consequence of the existence of the 2-fold degenerate symmetry broken states at large $\kappa$ alone. At large $\kappa$ we may form symmetric and antisymmetric linear combination of the two symmetry breaking ground states, so that they become eigenstates of $T$ with eigenvalues $t=0$ and $t=\pi$. It is simple to show that both (02) and (20) have eigenvalue $u=0$ with respect to the center-of-mass operator $U$. Consequently the newly formed linear combinations also have the same eigenvalue. Thus at large $\kappa$ we have a degenerate pair of ground states with quantum numbers $Q=(0,0)$ and $Q=(0, \pi)$. If adiabatic continuity exists, 


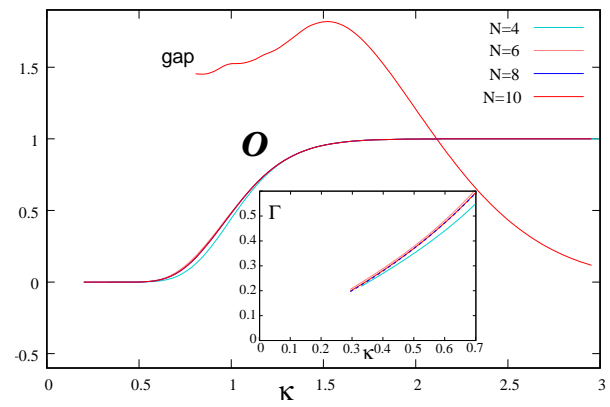

FIG. 2: Colored curves: the charge density wave order parameter $\mathcal{O}$ of the ground states in the zero center-of-mass sector for $N=4,6,8,10$ particles. These states are adiabatically connected to the (20)- and (02)-states in Fig 1(a) and (b). The ground state that is connected to the (11) state of Fig. $1(\mathrm{c})$ is free of symmetry breaking, hence has $\mathcal{O}=0$. The single curve labeled "gap" shows the energy gap for $N=10$ versus $\kappa$ for $\kappa>\kappa_{s d}$. Gap data is scaled down by a factor of 4 for convenience. Inset: The quantity $\Gamma=1 / \sqrt{-\log \mathcal{O}}$.

we must have ground states with these quantum numbers at any $\kappa$, including $\tilde{\kappa}$. Now let us consider applying the duality transformation to the $Q=(0, \pi)$ ground state at $\tilde{\kappa}$. As we explained earlier, this transforms it to a state $Q=(\pi, 0)$ at $\kappa$. Since the ground state energy at $\kappa$ and $\tilde{\kappa}$ is the same, this transformed state must have the same energy as the $Q=(0,0)$ and $Q=(0, \pi)$ ground states at $\kappa$. Thus we have generated a third orthogonal state degenerate with the $Q=(0,0),(0, \pi)$ ground state at the same value of $\kappa$. Consequently the ground state is at least three-fold degenerate. In the large $\kappa$ limit the $Q=(\pi, 0)$ state is the (11) ground state.

Adiabatic continuity Fig 2) displays the charge density wave order parameter $\mathcal{O}=\sum_{n}(-1)^{n} c_{n}^{\dagger} c_{n} / N$ associated with the two symmetry breaking ground states in the $u=0$ sector of Eq. (3). As explained above, these states are evolved from the (20) and (02) states at large $\kappa$. This is manifested by the fact that $|\mathcal{O}|$ becomes 1 , reflecting perfect order, in the large $\kappa$ limit. We note that the value of $\mathcal{O}$ converges remarkably fast with increasing system size (Fig. 2), and it seems hardly necessary to look at larger systems for this purpose. The order parameter seemingly vanishes around $\kappa \approx .5$, very similar to the behavior observed in the abelian case [1]. This could lead one to think that there is a phase transition near this point, below which the order parameter is zero in the $N \rightarrow \infty$ limit. However, a careful study of the order parameter at small $\kappa$ suggests otherwise. In the inset we plot the quantity $\Gamma$ which is related to the order parameter via $\mathcal{O}=\exp \left(-1 / \Gamma^{2}\right)$. Although numerical precision limits us to $\kappa \gtrsim .29$, we observe that the order parameter continues to display a rapidly converging, alternating behavior as a function of system size below $\kappa=.5$. The fact that $\Gamma$ converges to nonzero values as a function of particle number for all $\kappa$ value we studied clearly implies that for these $\kappa$ value the limiting value $(N \rightarrow \infty)$ of the order parameter is nonzero. Moreover, it seems likely that the limiting $\Gamma$-curve extrapolates into the origin. This implies that as $N \rightarrow \infty$ the order parameters associated with the evolved (02) and (20) states stay non-zero except at $\kappa=0$ (infinite $L_{1}$ ). This is fully consistent with our findings in the abelian case 1,18 ]. Thus the analysis based on the order parameter suggests that there is no phase transition. A new feature that occurs in the nonabelian case is the existence of a non-symmetry-breaking ground state, i.e, the descendent of the (11) state, at all values of $\kappa$. Due to the exponentially small order parameter in Fig.2, the distinction between the two kinds of ground states becomes academic as $\kappa$ decreases below 0.5 .

Now we demonstrate that the exponentially small order parameter at small $\kappa$ does not affect the robustness of the energy gap. Fig. 2 shows the energy gap for 10 particles as a function of $\kappa$. The data is truncated below the self-dual point $\kappa_{s d}=\sqrt{2 \pi / N}$ because this part of the data is just the dual image of that above $\kappa_{s d}$. In addition, due to the strong size dependence of $\kappa_{s d}$, we expect the data below $\kappa_{s d}$ to display a stronger size dependence, and hence to be less useful for the purpose of extrapolation. Although due to the limitation on particle number, our study of the gap is limited to $\kappa \gtrsim .8$, it is apparent that the energy gap does not decline as the order parameter becomes exponentially small. In addition to the above numerical evidence, there is an extra reason to believe that for the bosonic non-abelian state the gap cannot be caused by symmetry breaking, because there is a totally symmetric ground state at all values of $\kappa$. To summarize, both the numerical results for the order parameter and the energy gap are consistent with the notion of adiabatic continuity between small and large $\kappa$.

Quasiparticles Again, we first understand the quasiparticle and quasihole in the large $\kappa$ limit. The most naive way to produce a charge $\Delta Q=1$ state is to remove one boson from one of the ground states, say the (02), as shown in Fig.1(d). (Using the nomenclature of the electron, we regard the boson as negatively charged.) As for all the abelian cases, the positively charged defects cost zero energy. A closer inspection of Fig.1(d) reveals that such a defect is consisted of two domain walls (between the ground state in Fig.1(a) and 1(c)) placed at the closest distance. Since a string of 1's does not cost any additional energy, it is possible to separate the two domain walls, without injecting any extra charge, by converting an even sequence of 0202..02 into a string of 1's as shown in Fig.1(e). The two domain walls at the end of the string each carry a charge $\Delta Q=1 / 2$. Now let us consider removing a boson from the (11) ground state. By turning an odd string of 1's into $0202 \ldots .20$ as sown in Fig.1(f) two domain walls of charge $\Delta Q=1 / 2$ are produced. A similar procedure can lead to $\Delta Q=-1 / 2$, nonzero energy, anti-domain walls. Since there is adia- 
batic continuity we should be able to tune $\kappa$ down and let each of these domain walls evolve into the fractional charged quasiholes (delocalized in the $L_{1}$-direction) in the non-Abelian Hall liquid. We note that the quasiparticle/quasi-hole charge of $\Delta Q= \pm 1 / 2$, which follows naturally from our simple large $\kappa$ analysis, indeed agrees with the prediction in Ref. [9].

Next we consider four quasiholes. In Fig.1(g) and (h) two distinct four domain wall configurations (on top of the (02) ground state) are presented. These patterns are differentiated by the fact that in Fig.1(g)/(h) the middle string of 0,2 's are in phase/out of phase with those at the two ends. To transform panel (g) to (h) one has to move the 1 at the left end of the middle 0,2 string to the right end and shift the entire 0,2 string to the right. For far separated domain walls this move clearly involves many particles and is very non-local. Comparing Fig.1(g) and (h) one might regard the two middle domain walls as having different positions (a shift of one lattice constant). However this distinction will cease to exist if we let $\kappa$ decrease so that further range hopping can take place. In that case each of the domain wall discussed above will smear into wider and smoother transition region between different ground states. The width of the domain wall increases as $\kappa$ decreases. Although the tiny difference in the positions of the domain wall becomes immaterial as $\kappa$ decreases, the fact that in the case of infinitely-separated domain walls no finite particle and local moves can transform Fig.1(g) into (h) suggest that they will remain distinct. This results in a two-fold degeneracy for four quasihole state at fixed positions. Similarly for six quasiholes, we have three disconnected string of 1 's. In the gap between the first and second, and second and third strings (of 1's) we can again insert either (02) or (20) ground states. Thus for six qusiholes at fixed positions, we have a degeneracy of 4 . Continuing this line of argument, we conclude that in the presence of $2 n$ quasiholes, we have a $2^{n-1}$ fold degeneracy. Similar arguments can be carried out for the quasihole injection into the other two ground states. This is consistent with the prediction of the wavefunction analysis 13 .

The above arguments lead us to an interesting observation. There is an intricate difference between the twodomain-wall state in the (02) and (20) background and the (11) background. Let us first focus on the (11) background. Fig.1(i) and (j) represent two patterns of domain wall configurations differing by one lattice constant translation. Since we have removed a boson, which changes the filling factor away from 1, the $U$ eigenvalues of these two patterns are different. Hence they can not be mixed by further range hopping when we decrease $\kappa$ in Eq. (3). Now let us consider the (02) background. It is simple to convince oneself that due to the symmetry breaking and the periodic boundary condition we can only shift the two domain walls by even multiple lattice constants. Consequently there are twice as many two-domain-wall states in the (11) background as in the (02) and (20) background. Interestingly, the analysis of Greiter et al shows that for fixed quasihole positions, including all ground states, the two-quasihole-state is four-fold rather than three-fold degenerate. We believe that the extra degeneracy is due to the existence of twice as many two-domain wall states that can be added to the (11) ground state.

In conclusion, we have shown that the bosonic nonabelian Hall liquid is adiabatically connected to simple density patterns as the small dimension of the torus decreases. Together with our earlier result on the abelian Hall liquid [1, 4, 8], a powerful conclusion emerges. The paradigms of quantum number fractionalization in one dimension (polyacetylene) and two dimension (fractionally quantum Hall effect) are in fact equivalent.

Acknowledgement: In the process of preparing this manuscript, we become aware of a preprint by Bergholtz et al 14] on a closely related subject for the $\nu=1 / 2$ fermionic pfaffian state. DHL and AS are supported by DOE grant DE-AC03-76SF00098.

[1] A. Seidel, H. Fu , D.-H. Lee, J. M. Leinaas, J. Moore Phys. Rev. Lett. 95, 266405 (2005).

[2] W. P. Su, J. R. Schrieffer, A. J. Heeger, Phys. Rev. B 22, 2099 (1980)

[3] R. B. Laughlin, Phys. Rev. Lett. 50, 1395 (1983)

[4] E. J. Bergholtz and A. Karlhede, J. Stat. Mech. (2006) L04001

[5] D. H. Lee, J. M. Leinaas, Phys. Rev. Lett. 92, 096401 (2004)

[6] E.J. Bergholtz and A. Karlhede, Phys. Rev. Lett. 94, 26802 (2005).

[7] S. A. Trugman, S. Kivelson, Phys. Rev. B 31, 5280 (1985).

[8] E. H. Rezayi and F. D. M. Haldane, Phys. Rev. B, 50, 17199 (1994).

[9] G. Moore, N. Read, Nucl. Phys. B360, 362 (1991)

[10] M. Oshikawa, Phys. Rev. Lett. 84, 1535 (2000)

[11] M. Greiter, X.G. Wen, F. Wilczek Nucl. Phys. B374, 567 (1992)

[12] N. Read, E. Rezayi, Phys. Rev. B 54, 16864 (1996)

[13] C. Nayak and F. Wilczek, Nucl. Phys. B479, 529 (1996).

[14] E.J. Bergholtz et al, cond-mat/0604251 